\title{
Acessibilidade da População Negra ao Cuidado Oncológico no Brasil:Revisão Integrativa
}

doi: https://doi.org/10.32635/2176-9745.RBC.2019v65n4.453

\author{
Accessibility of Black Population to Oncological Care in Brazil: Integrative Review \\ Accesibilidad de la Población Negra a la Atención Oncológica en Brasil: Revisión Integrativa
}

Janaína Santos Paulista'; Paula Gonçalves Assunção²; Fernando Lopes Tavares de Lima ${ }^{3}$

Resumo

Introdução: Tem-se apontado a persistência de desigualdades raciais na acessibilidade a bens e serviços de saúde, estando o indivíduo com câncer inserido nesse contexto. Objetivo: Analisar os fatores que impactam a acessibilidade da populaçáo negra ao cuidado oncológico no Brasil. Método: Por meio de uma revisão integrativa da literatura, buscaram-se, nas bases PubMed, LILACS e Embase, artigos originais publicados em português, inglês e espanhol sem limite temporal. Resultados: Foram incluídos 13 artigos, analisados a partir do tipo de câncer abordado: câncer de mama, câncer ginecológico, câncer de próstata e câncer de cavidade oral. Conclusão: As desigualdades raciais e socioeconômicas refletem nas açóes de acessibilidade ao cuidado oncológico, maioritariamente na detecção precoce. Independentemente do tipo de câncer e das formas de diagnóstico e de tratamento, é inegável a dificuldade do paciente negro aos cuidados oncológicos por razóes de natureza social e econômica. Os profissionais de saúde deveriam assegurar o atendimento à população negra ao mesmo nível prestado aos demais grupos sociais para garantir e superar as barreiras de acesso aos bens e serviços de saúde.

Palavras-chave: Neoplasias; Saúde das Minorias Étnicas; Grupo com Ancestrais do Continente Africano; Acesso Universal aos Serviços de Saúde.

\begin{abstract}
Introduction: The persistence of racial inequalities in the access to health services has been pointed out and the individual with cancer is within this scenario. Objective: To analyze the factors that impact the accessibility of the black population to cancer care in Brazil. Method: Through an integrative literature review, it were searched original articles published in Portuguese, English and Spanish in the databases PubMed, LILACS and Embase without time limitation. Results: Thirteen articles were included and analyzed based in the type of cancer addressed: breast cancer, gynecological cancer, prostate cancer and oral cancer. Conclusion: The racial and socioeconomic inequalities reflect upon the actions of accessibility to cancer care, mostly in early detection. Regardless of the type of cancer and the forms of diagnosis and treatment, it is unquestionable the difficulty the afro-descendant population has to access oncological care because of social and economic issues. Healthcare providers must ensure the care to the afro-descendant population as much as they do with other social groups to overcome the barriers to access health services.

Key words: Neoplasms; Health of Ethnic Minorities; African Continent Ancestry Group; Universal Access to Health Care Services.
\end{abstract}

\section{Resumen}

Introducción: Se ha señalado la persistencia de las desigualdades racial e la accesibilidad a los bienes y servicios de salud, con el individuo con cáncer insertado en este contexto. Objetivo: Analizar los factores que afectan la accesibilidad de la población negra a la atención del cáncer en Brasil. Método: A través de una revisión bibliográfica integradora, se realizar búsquedas en las bases de datos PubMed, LILACS e Embase artículos originales publicados en portugués, inglés y español sin límite de tiempo. Resultados: Se incluyeron 13 artículos, analizado sen función del tipo de cáncer abordado: cáncer de mama, cáncer ginecológico, cáncer de próstata y cáncer de cavidad oral. Conclusión: Las desigualdades raciales y socioeconómicas se reflejan en las acciones de accesibilidad a la atención del cáncer, principalmente en la detección temprana. Independientemente del tipo de cáncer y las formas de diagnóstico y tratamiento, la dificultad del paciente negro en la atención del cáncer es innegable por razones sociales y económicas. Los profesionales de la salud deben garantizar la atención de la población negra al mismo nivel que otros grupos sociales para garantizar y superar las barreras de acceso a los bienes y servicios de salud.

Palabras clave: Neoplasias; Salud de las Minorías Étnicas; Grupo de Ascendencia Continental Africana; Acceso Universal a los Servicios de Salud.

\footnotetext{
${ }^{1}$ Egressa do Programa de Residência Multiprofissional em Oncologia do Instituto Nacional de Câncer José Alencar Gomes da Silva (INCA). Rio de Janeiro (RJ), Brasil. ORCID iD: https://orcid.org/0000-0002-2146-5259

${ }^{2}$ Egressa do Programa de Residência Multiprofissional em Oncologia do INCA. Rio de Janeiro (RJ), Brasil. ORCID iD: https://orcid.org/0000-0003-3764-2544

${ }^{3}$ Coordenação de Ensino do INCA. Rio de Janeiro (RJ), Brasil. ORCID iD: https://orcid.org/0000-0002-8618-7608

Endereço para correspondência: Janaína Santos Paulista. Rua Barata Ribeiro 502, apto. 705 - Copacabana. Rio de Janeiro (RJ), Brasil. CEP $22040-002$.

E-mail: naina_santos@yahoo.com.br
} 


\section{INTRODUÇÃO}

No Brasil, o Sistema Único de Saúde (SUS) reconhece a saúde como um direito a ser assegurado pelo Estado, estando fundamentado nos princípios da universalidade, equidade e integralidade. Ou seja, no âmbito legal, adota-se a concepçáo ampliada de saúde e a obrigatoriedade e a gratuidade do acesso aos bens e serviços de saúde à toda população brasileira ${ }^{1,2}$. Porém, são comuns as situaçóes que contrastam injustiça social e esses direitos de acesso e acessibilidade ${ }^{3}$.

Acesso e acessibilidade são conceitos complexos abordados de diversas formas, mas compreendidos como termos complementares. Entende-se por acesso a disponibilidade dos serviços de saúde do ponto de vista da oferta e, por acessibilidade, a possibilidade concreta de acessar aos serviços ou recursos ofertados ${ }^{4}$. Para Donabedian $^{5}$, a acessibilidade se relaciona também com a adequação dos profissionais e dos recursos tecnológicos utilizados diante da necessidade dos usuários 5 . Sua compreensão vai além da entrada no serviço de saúde, contemplando dimensóes socioeconômicas, geográficas, culturais e políticas, as quais podem constituir barreiras no atendimento dos usuários no SUS.

As iniquidades da acessibilidade em saúde são produzidas a partir de inúmeras determinaçóes, entre elas, diferentes modalidades de discriminação como raça/etnia, religião, gênero e sexualidade ${ }^{6}$. Entre esses elementos, as barreiras econômicas e étnico-raciais destacam-se na reprodução das iniquidades e intensificam a vulnerabilização dos diferentes grupos ${ }^{7}$.

A atual classificação do Instituto Brasileiro de Geografia e Estatística (IBGE), em relação ao quesito "cor ou raça", é dividida em: brancos, pardos, pretos, amarelos e indígenas. O negro é o somatório das populaçōes pardas e pretas e essa identificação é feita por autodeclaração. No Censo demográfico de 2010, declararam-se negros 97 milhôes de pessoas, enquanto 91 milhóes consideraram-se brancas. Apesar de ser maioria, os negros constituem a camada mais pobre da população .

Buscando enfrentar a inequidade relacionada à cor de pele, o Conselho Nacional de Saúde aprovou, de forma unânime, em 2006, a criação da Política Nacional de Atençâo Integral à Saúde da População Negra (PNSIPN). Nela, reconhecem-se as desigualdades raciais como fatores que interferem no processo saúde/doença/cuidado e morte. Apenas em 2009, a PNSIPN foi pactuada na Comissáo Intergestores Tripartide, sendo o racismo reconhecido como um fator que impacta a saúde ${ }^{9,10}$.

O racismo é considerado uma realidade ideológica que estrutura relaçóes sociais e concede superioridade de uma raça sobre as demais, propagando discriminação e preconceito racial por meio de atitudes racistas, conscientes ou inconscientes. Por ocorrer por meio de políticas sociais, o racismo institucional é visto como o fracasso coletivo de uma organização na promoção de serviços adequados às pessoas, por sua cor, cultura ou origem étnica, inviabilizando avanços efetivos na equidade das açôes ${ }^{11}$.

O racismo se manifesta pela dificuldade em reconhecer e abordar os determinantes sociais das condiçôes de saúde, na utilizaçáo desagregada de dados para orientar uma tomada de decisão, na definição de prioridades e alocação dos recursos, bem como na ausência de meios de coibiçấo de práticas racistas inadmissíveis ${ }^{10}$. Essa desigualdade também se manifesta no acesso aos serviços de atenção oncológica, mesmo diante de estratégias de combate ao racismo institucional e ações que beneficiem a equidade na saúde ${ }^{12}$.

A PNSIPN, ao reconhecer o racismo, as desigualdades étnico-raciais e o racismo institucional como determinantes sociais das condiçóes de saúde, elencou diversos objetivos a serem alcançados. Entre eles, garantir e ampliar o acesso da população negra do campo e da floresta e, em particular, das populaçôes quilombolas às açôes e aos serviços de saúde $e^{9,13}$.

Pesquisas sobre saúde e raça/etnia são escassas mesmo diante da necessidade de evidenciar o impacto do racismo na saúde ${ }^{14}$. Acredita-se que um dos motivos esteja na ausência do quesito cor em documentos e dados de saúde, o que não permite seu uso como variável de análise como indicador de raça/etnia.

A mortalidade negra, quando discutida, está relacionada às doenças cardiovasculares, causas externas, mortalidade infantil e materna e por HIV/aids entre mulheres ${ }^{9,13,15}$, sendo o câncer pouco abordado. Entretanto, estudos já apontam que, em mulheres negras, o diagnóstico para neoplasias de mama e do colo do útero é feito mais tardiamente em relaçáo às mulheres brancas ${ }^{14,16}$. Todavia, esses estudos são localizados, apontando a necessidade de análises mais globais ${ }^{9}, 10,13,15$.

Diante do exposto, acredita-se que ainda há uma carência de informaçôes sobre diferenças de acessibilidade e má qualidade de saúde que pode colaborar com o quadro da mortalidade dos negros. Questiona-se que, mesmo diante de políticas universais que possibilitam o enfrentamento aos processos de exclusão e buscam diminuir vulnerabilidades, estas não têm sido suficientes para a redução da disparidade racial em saúde. Assim, este estudo objetiva analisar, por meio de uma revisão de literatura, os fatores que impactam a acessibilidade da população negra ao cuidado em oncologia no Brasil.

\section{MÉTODO}

Trata-se de um estudo de revisão sistemática integrativa, que permite a síntese e a análise crítica do conhecimento científico sobre determinado tema ou questáo norteadora, contribuindo para a prática na saúde baseada em evidência $(\mathrm{PBE})^{17}$. 
Para sua elaboração, foram percorridas seis etapas ${ }^{18}$ : 1- identificação do tema e seleção da questão norteadora; 2- estabelecimento de critérios para inclusão e exclusão dos estudos; 3- definiçấo das informaçôes a serem extraídas dos estudos selecionados e sua categorização; 4- avaliação metodológica dos estudos incluídos; 5- interpretação dos resultados; 6- apresentação da revisão e síntese do conhecimento.

Na primeira etapa, foi elaborada a seguinte pergunta norteadora: Quais fatores que impactam a acessibilidade da população negra ao cuidado em oncologia no Brasil?

Foram incluídos estudos originais disponíveis nas bases PubMed, Literatura Latino-Americana e do Caribe em Ciências da Saúde (LILACS) e Embase. A busca foi realizada no período entre junho e setembro de 2018. Foram incluídos os trabalhos brasileiros que abordavam os aspectos relacionados à acessibilidade da população negra ao cuidado em oncologia (promoção, prevenção, tratamento e cuidados paliativos) publicados nos idiomas português, inglês e espanhol. Foram excluídos estudos que não abordaram a realidade da população negra especificamente no Brasil, estudos secundários, cartas-resposta, editoriais, dissertaçôes, teses, capítulos de livro, relatos de experiência, assim como aqueles indisponíveis na íntegra. Não foi estabelecido limite temporal das publicaçóes, a fim de abranger um maior número de trabalhos. Foram utilizados os descritores "African Continental Ancestry Group", "Neoplasms", "Health Services Accessibility" e "Brazil", associados por meio dos operados booleanos AND e OR.

Posteriormente à fase de inclusão dos trabalhos, foi realizada a extração das informaçôes referentes à relação entre a cor da pele negra e dados relacionados à acessibilidade às açóes de prevenção e controle do câncer, bem como aos índices de morbidade ou mortalidade dessa população por essa enfermidade.

Para análise da qualidade dos artigos incluídos, optou-se em aplicar dois instrumentos complementares: o instrumento adaptado do Critical Appraisal Skill Programme (CASP) e o da Agency for Healthcare and Research and Quality (AHRQ) ${ }^{19,20}$.

O CASP adaptado contempla dez itens a serem pontuados: 1) objetivo claro e justificado; 2) metodologia adequada; 3) apresentação e discussão dos procedimentos teóricos e metodológicos; 4) seleção adequada da amostra; 5) coleta de dado detalhada; 6) relaçáo entre pesquisador e pesquisados; 7) aspectos éticos preservados; 8) análise de dados rigorosa e fundamentada; 9) apresentação e discussão dos resultados; e 10) contribuiçôes, limitaçôes e indicaçôes de novas questôes de pesquisa. Para cada item, foi atribuído um valor de zero ou um, sendo o resultado a soma das pontuaçóes, cujo escore máximo é de dez pontos. Os artigos selecionados foram classificados conforme as pontuações: nível A - seis a dez pontos (boa qualidade metodológica e viés reduzido) ou nível B - um a cinco pontos (qualidade satisfatória, porém com risco de viés aumentado).

A AHRQ classifica estudos em sete níveis conforme o nível de evidência: I) revisão sistemática ou metanálise; II) ensaios clínicos randomizados; III) ensaios clínicos sem randomização; IV) coorte e caso-controle; V) revisôes sistemáticas de estudos descritivos e qualitativos; VI) único estudo descritivo ou qualitativo; e VII) opinião de autoridades e/ou relatório de comitês de especialidades.

\section{RESULTADOS}

Foram encontrados 203 trabalhos, sendo excluídos 43 por não abordarem a temática do estudo e quatro por serem duplicados. Além disso, 135 foram excluídos por não responderem à questão norteadora e oito por serem documentos oficiais e decretos. Assim, foram incluídos para análise 13 artigos, conforme o Fluxograma apresentado na Figura 1.

Os estudos foram publicados entre 2003 e 2014. Verificou-se que 11 trabalhos foram classificados como

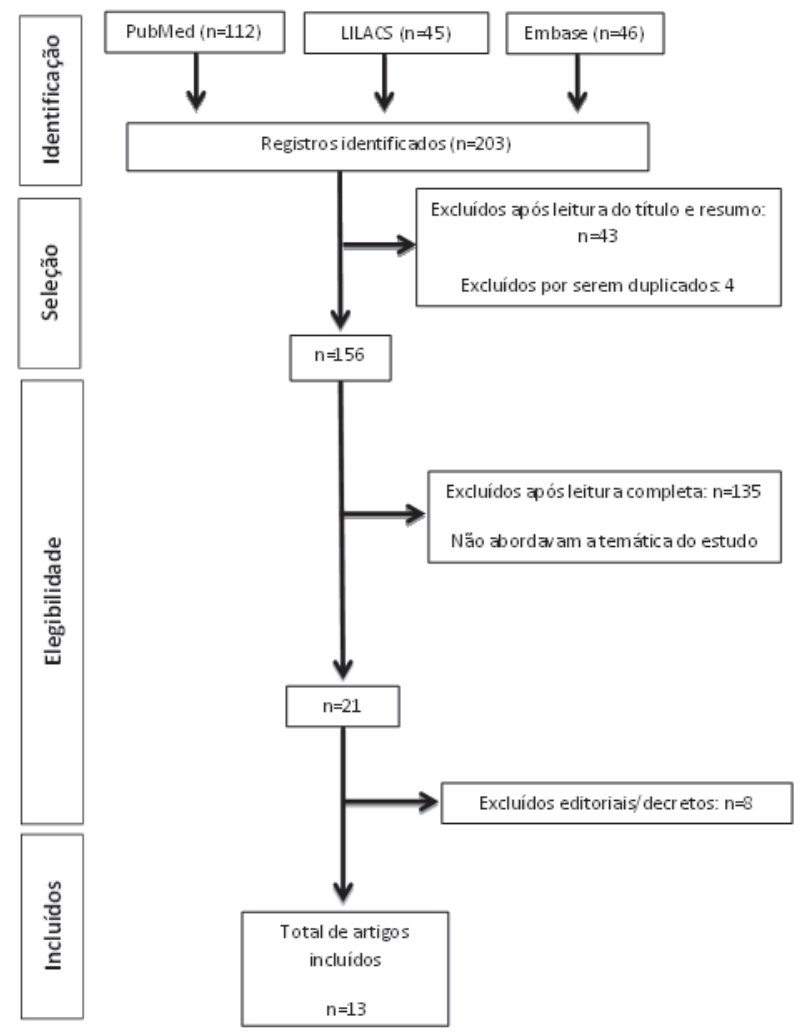

Figura 1. Fluxograma do processo de seleção dos artigos para a revisão de artigos (adaptado da Recomendação PRISMA) ${ }^{21}$ 
nível A no CASP adaptado, apresentando boa qualidade metodológica. Entretanto, pela AHRQ, verificou-se que 11 estudos foram de nível VI, em virtude do tipo de estudo realizado.
O Quadro 1 apresenta dados gerais sobre os artigos incluídos: autoria, ano de publicação, desenho do estudo, número de participantes (amostra), objetivos e nível de evidência segundo o CASP adaptado e a AHRQ.

Quadro 1. Descrição compilada e níveis de evidência, segundo o CASP adaptado e a AHRQ, Rio de Janeiro, 2018

\begin{tabular}{|c|c|c|c|c|}
\hline Autor/ano & $\begin{array}{l}\text { Desenho do } \\
\text { estudo }\end{array}$ & Amostra & Objetivo & $\begin{array}{l}\text { Evidência } \\
\text { CASP/AHRQ }\end{array}$ \\
\hline $\begin{array}{l}\text { Amorim et al., } \\
2006^{22}\end{array}$ & Transversal & $\begin{array}{c}290 \\
\text { mulheres }\end{array}$ & $\begin{array}{l}\text { Analisar a prevalência da não realização do } \\
\text { exame de Papanicolau segundo variáveis } \\
\text { socioeconômicas, demográficas e de } \\
\text { comportamentos relacionados à saúde, em } \\
\text { mulheres com } 40 \text { anos ou mais de idade, } \\
\text { residentes no Município Campinas, São Paulo, } \\
\text { Brasil }\end{array}$ & Nível A/ VI \\
\hline $\begin{array}{l}\text { Amorim et al., } \\
2008^{23}\end{array}$ & Transversal & $\begin{array}{c}290 \\
\text { mulheres }\end{array}$ & $\begin{array}{l}\text { Analisar a prevalência da não realização do } \\
\text { exame clínico das mamas e da mamografia } \\
\text { segundo variáveis socioeconômicas, } \\
\text { demográficas e de comportamentos } \\
\text { relacionados à saúde, em mulheres com } \\
40 \text { anos ou mais, residentes na cidade de } \\
\text { Campinas, São Paulo, Brasil }\end{array}$ & Nível A/ VI \\
\hline $\begin{array}{l}\text { Antunes et al., } \\
2013^{24}\end{array}$ & Transversal & $\begin{array}{c}8.505 \\
\text { pessoas }\end{array}$ & $\begin{array}{l}\text { Analisar tendências recentes da mortalidade } \\
\text { por câncer de cavidade oral, focalizando } \\
\text { diferenças quanto ao sexo e à raça }\end{array}$ & Nível A/ VI \\
\hline $\begin{array}{l}\text { Bairros et al., } \\
2011^{14}\end{array}$ & Transversal & $\begin{array}{c}2.030 \\
\text { mulheres }\end{array}$ & $\begin{array}{l}\text { Investigar o acesso de mulheres negras e } \\
\text { brancas aos exames de detecção precoce } \\
\text { de câncer de mama e do colo do útero } \\
\text { (citopatológico), em duas cidades no Sul do } \\
\text { Brasil }\end{array}$ & Nível A/ VI \\
\hline $\begin{array}{l}\text { Boclin et al., } \\
2013^{25}\end{array}$ & Coorte & $\begin{array}{c}1.733 \\
\text { mulheres }\end{array}$ & $\begin{array}{l}\text { Estimar as prevalências de história } \\
\text { autorrelatada de miomas uterinos, segundo } \\
\text { características demográficas, socioeconômicas } \\
\text { e de acesso a serviços de saúde }\end{array}$ & Nível B/ IV \\
\hline $\begin{array}{l}\text { Boclin et al., } \\
2014^{26}\end{array}$ & Coorte & $\begin{array}{c}1.475 \\
\text { mulheres }\end{array}$ & $\begin{array}{l}\text { Investigar se a posição socioeconômica ao } \\
\text { longo da vida relaciona-se com a associação } \\
\text { entre cor/raça e ocorrência de leiomiomas } \\
\text { uterinos }\end{array}$ & Nível B/ IV \\
\hline $\begin{array}{l}\text { Cesar et al., } \\
2003^{27}\end{array}$ & Transversal & $\begin{array}{c}1.302 \\
\text { mulheres }\end{array}$ & $\begin{array}{l}\text { Identificar a prevalência da não realização } \\
\text { de exame citopatológico entre mulheres de } \\
15 \text { a } 49 \text { anos em algum momento de suas } \\
\text { vidas, e possíveis fatores associados à sua não } \\
\text { realização }\end{array}$ & Nível A/ VI \\
\hline $\begin{array}{l}\text { Nardi et al., } \\
2012^{28}\end{array}$ & Transversal & $\begin{array}{c}1.915 \\
\text { homens }\end{array}$ & $\begin{array}{l}\text { Descrever as características epidemiológicas } \\
\text { e os padrões de atenção inicial ao câncer de } \\
\text { próstata em instituições públicas e privadas do } \\
\text { Estado de São Paulo, Brasil }\end{array}$ & Nível A/ VI \\
\hline $\begin{array}{l}\text { Oliveira et al., } \\
2014^{29}\end{array}$ & Transversal & $\begin{array}{c}348 \\
\text { mulheres }\end{array}$ & $\begin{array}{l}\text { Analisar os fatores associados à não realização } \\
\text { do exame de Papanicolau entre as mulheres } \\
\text { quilombolas residentes em Vitória da Conquista, } \\
\text { Bahia }\end{array}$ & Nível A/ VI \\
\hline
\end{tabular}


Quadro 1. continuação

\begin{tabular}{|c|c|c|c|c|}
\hline Autor/ano & $\begin{array}{l}\text { Desenho do } \\
\text { estudo }\end{array}$ & Amostra & Objetivo & $\begin{array}{l}\text { Evidência } \\
\text { CASP/AHRQ }\end{array}$ \\
\hline $\begin{array}{l}\text { Ozawa e } \\
\text { Marcopito, } \\
2011^{30}\end{array}$ & Transversal & $\begin{array}{l}969 \text { no } \\
\text { primeiro } \\
\text { inquérito e } \\
1.125 \text { no } \\
\text { segundo }\end{array}$ & $\begin{array}{l}\text { Comparar a cobertura da citologia } \\
\text { convencional de Papanicolau em mulheres } \\
\text { de } 15 \text { a } 59 \text { anos entre dois inquéritos } \\
\text { domiciliários, relacionando a alguns atributos } \\
\text { pessoais e à tendência da mortalidade por } \\
\text { câncer do colo do útero }\end{array}$ & Nível A/VI \\
\hline $\begin{array}{l}\text { Quadros et al., } \\
2004^{31}\end{array}$ & Transversal & $\begin{array}{l}1.730 \\
\text { mulheres }\end{array}$ & $\begin{array}{l}\text { Determinar a cobertura e o foco do } \\
\text { rastreamento do câncer do colo do útero } \\
\text { (exame de Papanicolau) em uma amostra de } \\
\text { base populacional no Brasil }\end{array}$ & Nível A/ VI \\
\hline $\begin{array}{l}\text { Souza et al., } \\
2013^{32}\end{array}$ & Transversal & $\begin{array}{c}1.349 \\
\text { homens }\end{array}$ & $\begin{array}{l}\text { Investigar fatores relacionados à etnia, nível } \\
\text { educacional e sua correlação com o estágio do } \\
\text { câncer de próstata e sua influência sobre os } \\
\text { resultados do tratamento }\end{array}$ & Nível A/ VI \\
\hline $\begin{array}{l}\text { Thuler et al., } \\
2014^{33}\end{array}$ & Transversal & $\begin{array}{c}37.638 \\
\text { casos }\end{array}$ & $\begin{array}{l}\text { Analisar os fatores determinantes do } \\
\text { diagnóstico em estádio avançado em mulheres } \\
\text { com câncer do colo do útero no Brasil }\end{array}$ & Nível A/ VI \\
\hline
\end{tabular}

Legendas: $\mathrm{CASP}=$ Critical Appraisal Skill Programme; $\mathrm{AHRQ}=$ Agency for Healthcare and Research and Quality.

\section{DISCUSSÃO}

Foram encontrados poucos estudos que abordaram a acessibilidade da população negra às açôes de prevenção e controle do câncer no país. Entretanto, trata-se de trabalhos recentes, sendo que nove foram publicados após a aprovação da PNSIPN, que pode ter sido indutora de produção científica sobre o tema.

Além disso, destacou-se que o racismo institucional não foi referido em nenhum estudo, assim como os fatores determinantes de acessibilidade à saúde vinculados diretamente ao profissional de saúde e à sua autopercepção são discutidos de forma superficial. A manutenção de uma discussão incipiente sobre o tema no país não contribui para sua superação e acaba por contribuir em restringir, mesmo que indiretamente, na capacidade de inclusão da população negra na sociedade e impedindo a construção de um país democrático com igualdade de oportunidades ${ }^{34}$.

Os estudos analisados abordaram quatro sítios tumorais que foram utilizados para a sequência temática dessa discussão: mama, colo do útero, próstata e cavidade oral.

\section{CÂNCER DE MAMA}

Amorim et al. ${ }^{23}$ analisaram os fatores relacionados à não realizaçáo da mamografia e do exame clínico das mamas como medidas de detecçáo precoce do câncer de mama, diferenciando pela cor de pele. Segundo a pesquisa, que incluiu em 290 mulheres com idade a partir de 40 anos, não realizar o exame clínico das mamas esteve ligado a não ter cônjuge e apresentar baixa renda. Por sua vez, não realizar a mamografia nos dois últimos anos que antecederam à entrevista foi associado às mulheres negras com mais de 70 anos e de baixa renda ${ }^{23}$.

Por sua vez, Bairros et $\mathrm{a}^{14}$. identificaram que a probabilidade de as mulheres não realizarem os exames citopatológico e de mama foi significantemente maior nas negras, mesmo após a análise ajustada por renda, escolaridade, classe econômica e idade.

A recomendação do Ministério da Saúde é de que se realize a mamografia bienal para mulheres na faixa etária de 50 a 69 anos como forma de rastreio e que aconteça o diagnóstico precoce baseado no tripé: população alerta aos sinais e sintomas suspeitos; profissionais de saúde capacitados na avaliação de casos suspeitos; e sistemas e serviços de saúde preparados para garantir a confirmação diagnóstica oportuna e com qualidade ${ }^{35}$.

Apesar de o rastreamento por intermédio da mamografia ser o principal meio de detecçáo da neoplasia maligna da mama, há grandes problemas de acessibilidade nacionalmente e que náo podem ser simplesmente atribuídos à falta de equipamentos. A má distribuição pelas Unidades da Federação, caracterizada especialmente pelas melhores condiçóes no Sul e Sudeste e piores nas outras Regiôes, é mais uma característica da manutenção da iniquidade no $\mathrm{SUS}^{36}$.

Considerando que o câncer de mama é o tumor mais frequente entre as mulheres no Brasil, excetuando 
os tumores de pele não melanoma ${ }^{35}$, é preocupante a escassez de estudos. Por se tratar do câncer mais incidente nas mulheres brasileiras, é fundamental que os gestores e profissionais de saúde discutam e estabeleçam medidas que garantam o acesso ao diagnóstico precoce para a população em vulnerabilidade, tendo em vista que ele é determinante no prognóstico. Considerando o princípio da equidade, essas medidas devem considerar esses grupos mais vulneráveis como foco de priorização, como a populaçáo negra.

\section{CÂNCER GINECOLÓGICO}

O principal objetivo dos oito trabalhos ${ }^{22,25-27,29-31,33}$ que abordaram o câncer ginecológico foi analisar os fatores associados à não realização do exame citopatológico. Mais uma vez, as iniquidades de saúde geradas pela cor, idade, renda, nível educacional e ausência de um cônjuge foram relevantes e relacionadas a elementos locais. Além disso, fatores capacitantes para a acessibilidade à saúde ${ }^{37}$, como renda, cobertura securitária pública ou privada e oferta de serviços, foram expressivos na análise desses artigos.

O maior número de trabalhos sobre o câncer ginecológico pode estar relacionado com a priorização na agenda estatal. O Ministério da Saúde recomenda nacionalmente o exame citopatológico em mulheres assintomáticas com idade entre 25 anos e 64 anos, a cada três anos, após apresentar dois exames consecutivos normais. Além disso, desde 2014, está disponível pela rede pública a vacina tetravalente do HPV para meninas de 9 a 13 anos, estendida para os meninos de 11 a 13 anos a partir de 2017. Mesmo diante de formas de rastreamento e detecção precoce, as mulheres ainda apresentam estágios avançados da doença que podem limitar possibilidade de cura $^{35}$.

A não realização do exame de Papanicolaou foi significativamente mais frequente nas mulheres com escolaridade de até quatro anos, que se autorreferiram negras e com idade entre 40 e 59 anos. A maior prevalência de não realização do exame de Papanicolaou relacionada à raça persistiu mesmo após ajuste para idade e escolaridade, apontando a existência de desigualdade racial quanto ao acesso ao exame de citologia oncótica cervical ${ }^{22}$.

Em outro estudo, identificou-se que mulheres de cor negra, de menor idade, renda familiar e escolaridade, que não estavam vivendo com companheiro no momento da entrevista e que tiveram o primeiro parto com 25 anos ou mais de idade, apresentaram razóes de prevalências significativamente maiores à não realização de exame citopatológico para detecçáo do câncer do colo uterino em relação às demais mulheres. A relação entre a cor da pele e a realização do exame foi confirmada em análise multivariada, encontrando-se uma razão de prevalência de 1,3 (1,2-1,4) maior à náo prevenção do câncer do colo uterino em relaçáo àquelas de cor branca ${ }^{27}$.

A relação entre a discriminação racial e as atividades do programa de detecção precoce do câncer do colo foram bastante destacadas pelo estudo de Amorim et al..$^{22}$. Os autores relatam que durante o ciclo gravídico-puerperal também há discriminação, visto que mulheres negras e pardas têm menor acesso ao pré-natal, dificuldade no atendimento em maternidade e menor analgesia durante o parto ${ }^{22}$. Apesar da abordagem do tema, não foram apresentadas possíveis resoluçôes, fazendo com que o estudo se limitasse ao âmbito descritivo do problema. Para Werneck ${ }^{13}$, o racismo tem relação com as condiçóes em que a pessoa nasce, com sua trajetória familiar e individual, condiçôes de vida e moradia, condições de trabalho, emprego, renda e de acesso às informaçôes e aos bens e serviços, não podendo ser ignorado.

De uma forma geral, os motivos da ausência de exame Papanicolau são compreendidos como multifatoriais, estando associados, principalmente, à compreensão de que é um exame desnecessário e constrangedor, à escassez de conhecimento sobre seus objetivos e à dificuldade de acesso para realizá-lo ${ }^{22}$. Não existem estudos que verificam essas razões especificamente para a população negra, o que dificulta a adoção de medidas mais específicas a essa população.

Dados semelhantes em relação às características sociodemográficas foram encontrados no estudo realizado no extremo Sul do país. Nesse caso, a rede de serviços é abundante, sendo ofertada em todos os postos de saúde da rede pública municipal à pequena distância dos usuários, não ocorrendo dificuldade de acessibilidade para a populaçáo. Entretanto, 57\% das entrevistadas em idade fértil nunca realizaram um único exame citopatológico ${ }^{27}$. Entre as barreiras apontadas, destacam-se a falta de conhecimento quanto aos fatores de risco, desconhecimento quanto ao tipo de exame e/ou sua utilidade, concepção de fatalidade da doença, a não priorização frente a outras necessidades, a oposição do marido/companheiro, o tabu por se tratar de um exame pélvico, o longo tempo para obter o resultado e a ideia de que se trata de um exame caro ${ }^{27}$.

Os detalhes sobre a cobertura da realizaçáo do exame preventivo foram abordados em três estudos ${ }^{30,31,33}$. Em estudo realizado em dois momentos distintos, a associação direta entre acessibilidade e políticas públicas foi apontada por meio da análise da cobertura do exame preventivo. No primeiro momento em que o inquérito foi realizado (1980), não havia a consolidação de um sistema de saúde com equidade. No segundo momento (2001), a população já se beneficiava com a implantação da Constituição de 1988, com Norma Operacional Básica (NOB) de 1996 e 
com a Norma Operacional de Assistência à Saúde (NOAS) de 2001. Identificou-se que a cobertura do exame em 2001 foi $80 \%$ superior quando comparada a $1987^{30}$.

Foi expressivo, neste estudo, o aumento de acessibilidade da população vulnerável; ou seja, mulheres negras, solteiras, de baixo nível educacional, após a reorganização da rede de saúde municipal pelo SUS. Esse dado corrobora o de outros autores, que evidenciaram a existência do SUS como o principal responsável pela realização do Papanicolau nesses grupos ${ }^{22,31,33}$. Todavia, mesmo com esse aumento, questôes de oferta e organizaçáo dos serviços de saúde ainda se compóem como barreiras e geram consequências no agravamento do estádio da doença ${ }^{33}$, como a falta de médicos ou vagas em consultas ${ }^{30}$.

Ainda no contexto dos tumores ginecológicos, dois estudos trouxeram dados sobre os miomas uterinos (MU), que são neoplasias benignas monoclonais de crescimento lento consideradas como os tumores mais comuns do sistema reprodutor feminino ${ }^{25,26}$. O MU é mais frequente em mulheres negras e entre aquelas expostas a fatores associados ao aumento de hormônios ovarianos (estrogênio e progesterona). É responsável pela queda na qualidade de vida de mulheres jovens em idade reprodutiva, principalmente em razão de cirurgias mutiladoras (histerectomia) ${ }^{25,26}$. No Brasil, mesmo com políticas governamentais que buscam ampliar, qualificar e humanizar a atenção integral à saúde da mulher, considerando especificidades étnico-raciais, ainda há escassez de dados sociodemográficos e de acesso a serviços de saúde para uma análise mais apurada.

Como variáveis marcadoras de acesso e utilização de serviços de saúde, nos dois estudos, foi considerada a realização do Papanicolau, além da origem do atendimento; ou seja, se os entrevistados possuíam ou não plano de saúde ${ }^{25,26}$. Os resultados vão ao encontro com os demais desta revisão, salientando mais uma vez a vulnerabilidade da mulher negra principalmente com baixa escolaridade e seu maior risco de apresentar MU. Um dos estudos investigou ainda se a posição socioeconômica se relaciona com ocorrência de $\mathrm{MU}$ e tal fato não foi constatado.

A focalização dos programas preventivos para mulheres de maior idade acaba náo priorizando outros fatores de risco, como a cor da pele. Como as prioridades são elencadas exclusivamente baseadas na idade, mulheres negras jovens, que possuem mais chances de apresentarem o MU, não são incluídas nesses programas. Ignora-se o fato de terem menor escolaridade, maior vulnerabilizaçấo social, serem mais vítimas de violência sexual e mais expostas à sexualizaçáo precoce, o que pode ser reflexo do racismo estrutural muito presente em nossa cultura.

\section{CÂNCER DE PRÓSTATA}

O câncer de próstata foi discutido em dois trabalhos com abordagens diferentes ${ }^{28,32}$. Um dos estudos abordou o atendimento ofertado a homens com neoplasia de próstata em instituiçóes particulares e públicas ${ }^{28}$. $\mathrm{O}$ outro analisou a raça e o nível educacional como fatores predisponentes em relaçáo ao diagnóstico e ao tratamento no câncer de próstata $^{32}$.

Por meio de um estudo transversal com 1.915 participantes atendidos pelo setor público (1.026) e privado (889), dados biológicos e sociodemográficos foram comparados ${ }^{28}$. Os que tiveram o primeiro atendimento em instituiçóes públicas eram negros, tinham maior idade, com antígeno sérico específico da próstata (PSA) elevado e maior probabilidade de metástase. Contudo, foram menos propensos a sofrer prostatectomia quando comparados aqueles que receberam atendimento particular ${ }^{28}$. Considerando que o câncer de próstata possui altas taxas de sobrediagnóstico e sobretratamento ${ }^{38}$, essa disparidade da assistência em saúde poderia estar refletindo positivamente para os negros, sendo necessários mais estudos que abordem esse fator para uma compreensão mais completa desse fenômeno.

Por sua vez, em estudo ${ }^{32}$ realizado retrospectivamente com 1.349 prontuários, avaliou-se a probabilidade de o paciente apresentar metástase vinculada ao nível de escolaridade e raça. A análise confirmou que os níveis educacionais e a cor da pele são fatores de risco independentes para metástases a distância no momento do diagnóstico. Pacientes com uma cor de pele negra apresentaram aumento de $300 \%$ em metástases agressivas em comparação com pacientes de outras cores de pele.

Segundo os autores, estudos internacionais mostram que fatores relacionados à falta de educação por si só náo podem explicar os resultados do tratamento associados a esse grupo. É possível que fatores genéticos inerentes a essa população, incluindo a expressão de genes relacionados à atividade androgênica, afetem a apresentação do câncer de próstata. Esses fatores podem explicar a maior incidência de câncer de próstata e piores resultados de tratamento observados nesse grupo, excluindo o impacto de efeitos socioeconômicos ${ }^{32}$. No Brasil, onde os níveis socioeconômico e educacional estão diretamente relacionados à raça, a interação entre essas variáveis pode ter um impacto ainda maior nos resultados da doença.

Ambos os estudos identificaram que os negros com câncer de próstata possuíam quadros mais avançados da doença no início do tratamento, o que pode resultar em um pior prognóstico. Porém, diante do pequeno número de trabalhos encontrados, fica destacada a necessidade do desenvolvimento de mais estudos que analisem a relação 
da raça e essa enfermidade, tendo em vista que se trata do câncer mais comum em homens no país ${ }^{35}$.

\section{CÂNCER DE CAVIDADE ORAL}

Em Sáo Paulo, um único estudo investigou as tendências de mortalidade de câncer de cavidade oral em relação a gênero e raça. Todos que residem na capital do Estado que faleceram por essa neoplasia foram inseridos na pesquisa e as estimativas do Censo foram utilizadas para estratificar o sexo (masculino e feminino) e os grupos raciais (brancos e negros) para análise $e^{24}$.

Os achados apontaram que a mortalidade por câncer oral aumentou nas mulheres em relação aos homens e duplicou entre os negros. Apesar de esse aumento ser um problema de saúde pública, pesquisas nacionais permanecem avaliando fatores de risco sem inserir a raça como categoria analítica ${ }^{24}$.

Sabe-se que o consumo de álcool possui maior prevalência em camadas socioeconômicas mais baixas e, assim como o tabagismo, relaciona-se com um menor nível educacional. Esses fatores explicam o aumento da mortalidade em negros que se concentram em áreas periféricas de São Paulo, evidenciando assim a importância de uma análise que precisa ir além base biológica e que passe a considerar o peso dos estratos sociais. Negros usam menos os serviços preventivos do que os brancos e estão sujeitos a um acesso diferencial por racismo institucional, o que pode explicar o aumento da mortalidade. Ignorar essa análise é um fator que gera resultados negativos na acessibilidade ao cuidado ${ }^{24,39}$.

As principais limitaçóes deste artigo são o pequeno número de trabalhos incluídos, excetuando-se o câncer do colo do útero, e as limitaçóes dos desenhos desses estudos. Por isso, destaca-se a necessidade da realizaçáo de mais estudos originais que abordem as iniquidades da acessibilidade às açôes de prevenção e controle do câncer no país para a populaçáo negra.

\section{CONCLUSÃO}

As desigualdades raciais e socioeconômicas refletem nas açóes de acessibilidade ao cuidado oncológico, maioritariamente na detecção precoce. Independentemente do tipo de câncer e das formas de diagnóstico e de tratamento, é inegável a dificuldade do paciente negro aos cuidados oncológicos por razóes de natureza social e econômica. Os profissionais de saúde deveriam assegurar o atendimento à população negra ao mesmo nível prestado aos demais grupos sociais para garantir e superar as barreiras de acesso aos bens e serviços de saúde.

Algumas barreiras de acessibilidade são consideradas supostamente intransponíveis, como a idade e a raça, entretanto as dificuldades relacionadas à acesso, transporte, cultura, política e serviços são passíveis de superação e/ou decisão por parte dos gestores e profissionais.

Cabe ponderar que o acesso a recursos tecnológicos, questôes culturais e de religiáo não foram abordados como barreiras para a população negra nos estudos analisados, mesmo sendo considerados determinantes estruturais de iniquidades em saúde.

Por fim, vale destacar que a identificaçáo e a classificação do quesito "cor" não podem ser apenas uma escolha pessoal do pesquisador, pois podem gerar viés nos resultados e direcionar açóes prioritárias de saúde que sejam desfavoráveis aos vulneráveis. Há uma necessidade indiscutível de o profissional de saúde estender seu olhar prioritário para a população negra assim como já faz, rotineiramente, com crianças e idosos, transpondo barreiras de acessibilidade a bens e serviços de saúde.

\section{CONTRIBUIÇÕES}

Janaína Santos Paulista e Fernando Lopes Tavares de Lima participaram da concepção, análise, interpretação dos dados, revisão crítica com contribuição intelectual e aprovação final para publicação. Paula Gonçalves Assunção participou da concepção, da interpretação dos dados, da redação e revisão crítica com contribuição intelectual e aprovação final para publicação.

\section{DECLARAÇÃO DE CONFLITO DE INTERESSES}

Nada a declarar.

\section{FONTES DE FINANCIAMENTO}

Não há.

\section{REFERÊNCIAS}

1. Ministério da Saúde (BR), Secretaria Executiva. Sistema Único de Saúde (SUS): princípios e conquistas [Internet]. Brasília (DF): Ministério da Saúde; 2000. [acesso 2019 set. 17]. Disponível em: http://bvsms.saude.gov.br/bvs/ publicacoes/sus_principios.pdf

2. Brasil. Constituição (1988). Constituição da República Federativa do Brasil [Internet]. Brasília, (DF): Senado Federal; 1988. [acesso 2019 set. 17]. Disponível em: http://www.planalto.gov.br/ccivil_03/constituicao/ constituicao.htm

3. Ministério da Saúde (BR), Ministério da Previdência e Assistência Social. Relatório final [Internet]. 8a Conferência Nacional de Saúde; 1986 mar.17- 21; Brasília (DF): Conselho Nacional de Saúde; 1986. [acesso 2019 set. 18]. Disponível em: http://bvsms.saude.gov. br/bvs/publicacoes/8_conferencia_nacional_saude_. relatorio_final.pdf 
4. Travassos C, Martins M. Uma revisão sobre os conceitos de acesso e utilização de serviços de saúde. Cad Saude Publica. 2004;20(Suppl. 2):S190-S198. doi: http:// dx.doi.org/10.1590/S0102-311X2004000800014

5. Donabedian A. Aspects of medical care administration: specifying requirements for health care. Cambridge: Harvard University Press; 1973.

6. Braveman P. Health disparities and health equity: concepts and measurement. Annu Rev Public Health. 2006; 27:167-94. doi: https://doi.org/10.1146/annurev. publhealth.27.021405.102103

7. Trad LAB, Castellanos MEP, Guimarães MCS. Acessibilidade à atenção básica a famílias negras em bairro popular de Salvador, Brasil. Rev Saude Publica. 2012;46(6):1007-13. doi: http://dx.doi.org/10.1590/ S0034-89102012000600010

8. Anjos G. A questão cor ou raça nos censos nacionais. Indic Econ FEE [Internet]. 2013 [acesso 2019 set. 18];41(1):103-118. Disponível em: https:// revistas.fee.tche.br/index.php/indicadores/article/ viewFile/2934/3163

9. Fundação Nacional de Saúde (BR). Saúde da população negra no Brasil: contribuiçôes para a promoção de equidade [Internet]. Brasília (DF): FUNASA; 2005. [acesso 2019 set. 18]. Disponível em: http://bvsms.saude. gov.br/bvs/pop_negra/pdf/saudepopneg.pdf

10. Batista LE, Monteiro RB, Medeiros RA. Iniquidades raciais e saúde: o ciclo da política de saúde da população negra. Saude Debate. 2013; 37(99): 681-90. doi: http:// dx.doi.org/10.1590/S0103-11042013000400016

11. López LC. O conceito de racismo institucional: aplicaçóes no campo da saúde. Interface Comun Saúde Educ. 2012;16(40):121-34. doi: http://dx.doi.org/10.1590/ S1414-32832012005000004

12. Assis ES, Lima RCM. Serviço social na saúde e questão racial: análise preliminar do acesso da populaçáo negra à atenção oncológica. In: Instituto Nacional de Câncer José Alencar Gomes da Silva. Diálogos em saúde pública e serviço social: a experiência do assistente social em oncologia [Internet]. Rio de Janeiro: INCA; 2018. [acesso 2019 nov. 25]. Disponível em: https://www.inca.gov.br/ sites/ufu.sti.inca.local/files//media/document//dialogosem-saude-publica-completo.pdf

13. Werneck J. Racismo institucional e saúde da população negra. Saude Soc. 2016;25(3):535-49. doi: http://dx.doi. org/10.1590/s0104-129020162610

14. Bairros FS, Meneghel SN, Dias-da-Costa JS, et al. Racial inequalities in access to women's health care in southern Brazil. Cad Saude Publica. 2011;27(12):2364-72. doi: http://dx.doi.org/10.1590/ S0102-311X2011001200008

15. Batista LE, Werneck J, Lopes F, organizadores. Saúde da população negra [Internet]. 2. ed. Brasília (DF): ABPN; 2012. [acesso 2019 out. 12]. (Coleção negras e negros: pesquisas e debates). Disponível em: http://bvsms.saude. gov.br/bvs/publicacoes/saude_populacao_negra.pdf

16. Lucena LT, Zãn DG, Crispim PTB, et al. Fatores que influenciam a realização do exame preventivo do câncer cérvico-uterino em Porto Velho, Estado de Rondônia, Brasil. Rev Pan-Amaz Saúde. 2011;2(2):45-50. doi: http://dx.doi.org/10.5123/ S2176-62232011000200007

17. Callahan JL. Constructing a manuscript: distinguishing integrative literature reviews and conceptual and theory articles. Hum Resour Dev ver [Internet]. 2010 [acesso 2019 Oct. 12];9(3):300-4. Disponível em: https:// journals.sagepub.com/pb-assets/cmscontent/HRD/ Callahan.pdf

18. Mendes KDS, Silveira RCCP, Galvão CM. Revisão integrativa: método de pesquisa para a incorporação de evidências na saúde e na enfermagem. Texto ContextoEnferm. 2008;17(4):758-64. doi: http://dx.doi. org/10.1590/S0104-07072008000400018

19. CASP: Critical Appraisal Skills Programme [Internet]. Oxford: Critical Appraisal Skills Programme; c2018. CASP Checklists; [2013]. [cited 2019 Dec. 9]. Available from: http://www.casp-uk.net/casp-tools-checklists

20- Galvão CM. Níveis de evidência. Acta Paul Enferm. 2006;19(2):V. doi: http://dx.doi.org/10.1590/S010321002006000200001

21. Galvão TF, Pansani TSA, Harrad D. Principais itens para relatar revisóes sistemáticas e meta-análises: a recomendação PRISMA. Epidemiol Serv Saude. 2015;24(2):335-42. doi: http://dx.doi.org/10.5123/ S1679-49742015000200017

22. Amorim VMSL, Barros MBA, César CLG, et al. Fatores associados à não realizaçáo do exame de Papanicolaou: um estudo de base populacional no Município de Campinas, São Paulo, Brasil. Cad Saude Publica. 2006;22(11): 2329-38. doi: http://dx.doi.org/10.1590/ S0102-311X2006001100007

23. Amorim VMSL, Barros MBA, César CLG, et al. Fatores associados a não realização da mamografia e do exame clínico das mamas: um estudo de base populacional em Campinas, São Paulo, Brasil. Cad Saude Publica. 2008;24(11):2623-32. doi: http://dx.doi.org/10.1590/ S0102-311X2008001100017

24. Antunes JLF, Toporcov TN, Biazevic MGH, et al. Gender and racial inequalities in trends of oral cancer mortality in Sao Paulo, Brazil. Rev Saude Publica. 2013;47(3):470-8. doi: http://dx.doi.org/10.1590/ S0034-8910.2013047003724

25. Boclin KLS, Faerstein E. Prevalence of self-reported medical diagnosis of uterine leiomyomas in a Brazilian population: demographic and socioeconomic patterns in the Pro-Saúde Study. Rev Bras Epidemiol. 2013;16(2):301-13. doi: http://dx.doi.org/10.1590/ S1415-790X2013000200007 
26. Boclin KLI, Faerstein E, Szklo M. Does life-course socioeconomic position influence racial inequalities in the occurrence of uterine leiomyoma? Evidence from the pró-saúde study. Cad Saude Publica. 2014;30(2):305-17. doi: http://dx.doi.org/10.1590/0102-311X00025413

27. Cesar JA, Horta BL, Gomes G, et al. Factors associated with non-participation in screening for cervical cancer in Southern Brazil. Cad Saude Publica. 2003;19(5):1365-72. doi: http://dx.doi.org/10.1590/ S0102-311X2003000500014

28. Nardi AC, Reis RB, Zequi SC, et al. Comparison of the epidemiologic features and patterns of initial care for prostate cancer between public and private institutions: a survey by the Brazilian Society of Urology. Int Braz J Urol. 2012;38(2):155-164. doi: http://dx.doi. org/10.1590/S1677-55382012000200003

29. Oliveira MV, Guimarães MDC, França EB. Fatores associados a náo realização de Papanicolau em mulheres quilombolas. Cienc Saude Colet. 2014;19(11):453544. doi: http://dx.doi.org/10.1590/1413812320141911.15642013

30. Ozawa C, Marcopito LF. Teste de papanicolaou: cobertura em dois inquéritos domiciliários realizados no município de São Paulo em 1987 e em 2001-2002. Rev Bras Ginecol Obstet. 2011;33(5):238-45. doi: http:// dx.doi.org/10.1590/S0100-72032011000500006

31. Quadros CAT, Victora CG, Costa JSD. Coverage and focus of a cervical cancer prevention program in southern Brazil. Rev Panam Salud Publica [Internet]. 2004 [acesso 2019 Oct. 10];16(4):223-32. Disponível em: https:// scielosp.org/pdf/rpsp/2004.v16n4/223-232/en

32. Souza ABC, Guedes HG, Oliveira VCB, et al. High incidence of prostate cancer metastasis in Afro-Brazilian men with low educational levels: a retrospective observational study. BMC Public Health. 2013;13:537. doi: https://doi.org/10.1186/1471-2458-13-537

33. Thuler LCS, Aguiar SS, Bergmann A. Determinantes do diagnóstico em estadio avançado do câncer do colo do útero no Brasil. Rev Bras Ginecol Obstet. 2014;36(6):237-43. doi: http://dx.doi.org/10.1590/ S0100-720320140005010

34. Heringer R. Desigualdades raciais no Brasil: síntese de indicadores e desafios no campo das políticas públicas. Cad Saude Publica. 2002;18 (Suppl.):S57-S65. doi: http://dx.doi.org/10.1590/S0102-311X2002000700007

35. Instituto Nacional do Câncer José Gomes Alencar. Estimativa 2018: incidência de câncer no Brasil [Internet]. Rio de Janeiro: INCA; 2018. [acesso 2019 out. 10]. Disponível em: https:/www.inca.gov.br/sites/ ufu.sti.inca.local/files//media/document//estimativaincidencia-de-cancer-no-brasil-2018.pdf

36. Xavier, DR et al. Cobertura de mamografias, alocação e uso de equipamentos nas regióes de saúde. Saude Debate. 2016;40(110):20-35. doi: http://dx.doi. org/10.1590/0103-1104201611002
37. Andersen R, Newman JF. Societal and individual determinants of medical care utilization in the United States. Milbank Q. 2005;83(4):e1-e28. doi: http:// dx.doi.org/10.1111/j.1468-0009.2005.00428.x

38. Delpierre C, Lamy S, Kelly-Irving M, et al. Life expectancy estimates as a key factor in over-treatment: the case of prostate cancer. Cancer Epidemiol. 2013;37(4):462-8. doi: https://doi.org/10.1016/j.canep.2013.03.014

39. Wünsch Filho V, Mirra AP, López RVM, et al. Tabagismo e câncer no Brasil: evidências e perspectivas. Rev Bras Epidemiol. 2010;13(2):175-87. doi: http://dx.doi. org/10.1590/S1415-790X2010000200001

Recebido em 9/9/2019 Aprovado em 13/12/2019 